\title{
Improving Digital Reading Experiences With Mood-Based Content Selection
}

Wim Verhavert

iMinds, CUO, Social Spaces,

KU Leuven

Parkstraat 45 bus 3605 ,

B-3000 Leuven, Belgium

wim.verhavert@soc.kuleuven.be

Jeroen Vanattenhoven

iMinds, CUO, Social Spaces,

KU Leuven

Parkstraat 45 bus 3605 ,

B-3000 Leuven, Belgium

jeroen.vanattenhoven@soc.kuleuven.be

Dirk De Grooff

iMinds, CUO, Social Spaces,

KU Leuven

Parkstraat 45 bus 3605 ,

B-3000 Leuven, Belgium

dirk.degrooff@soc.kuleuven.be

Copyright is held by the author/owner(s)

CHI 2013 Extended Abstracts, April 27-May 2, 2013, Paris,

France.

ACM 978-1-4503-1952-2/13/04.

\begin{abstract}
Many present-day reading platforms for news consumption use topic selection and reading histories to filter reading content and feed the various recommender systems. In an attempt to overcome the deficiencies of this approach, we suggest an alternative: mood-based content selection with personalizations, based on the reading context (time and place). In this work, we conducted contextual inquiry sessions and designed an interactive prototype with iterative user feedback.
\end{abstract}

\section{Author Keywords \\ Content selection;tablets;e-reading; \\ personalization;context awareness}

\section{ACM Classification Keywords}

H.5.2 [Interfaces]: Prototyping.

\section{Introduction}

The research described in this document is conducted as part of the Smarter Media in Flanders (SMIF) project. In this project, all major publishers of Flanders were investigating how they could join forces using a centralized cloud infrastructure. Our research, in particular, intended to clarify how this vast amount of content (text, images and video) could be displayed to the end user of the system, i.e. the reader. More specifically, 
we were asked to explore new ways of displaying heterogeneous types of reading content, stemming from a multitude of news content providers, in an efficient and effective way to a single reader.

Many present day applications address this challenge by letting the reader select and prioritize keywords and topics. Examples of these are Flipboard [5], Prismatic [9], Zite [10] and Google Currents [4]. Our own experience with these systems showed that topic selection alone falls short in several respects. The results of the content selection are often not aligned with the current reading needs and the selection of topics and keywords tends to be tedious and is frequently ambiguous. The rationale of the present research should be read against the background of these deficiencies. By seeking alternative ways to display, structure and personalize a vast amount of different content sources, we aimed to improve the readers ease to use the data content, and thus ultimately enhance their reading experience.

\section{Method}

Considering the exploratory nature of our research, we employed a contextual inquiry technique [2] to understand how users currently read on digital devices. This method allows for uninterrupted observation periods augmented with on-the-spot discussions and storytelling. Having both paper and digital artifacts close-by prevented over-generalization and abstract reasoning.

\section{Participants}

As digital reading is still embryonic in Flanders, we explicitly recruited participants with an early adopter profile. Experience and expertise with reading on digital devices was deemed essential for our research. In order to investigate future digital reading platforms, we wanted to explore whether and how the reading habits of this group of experts have changed throughout time and whether any trends could be discovered across participants.

Using Twitter and a blog dedicated to e-reading (http://ereading.posterous.com) we recruited 7 participants $(n=7)$ for in-depth sessions. Albeit we strived for a mixed case selection in terms of age, gender, etc., our audience proved to be very uniform. No significant differences in reading strategies and habits could be noticed among the different participants. Further research is needed to verify whether these findings, collected among a small group of e-readers, in a particular setting as Flanders, can be extrapolated to other settings and audiences.

\section{Procedure}

First, we asked our participants to read a traditional hardcopy newspaper and a paper magazine. The research team provided these paper artifacts to them, in order to prevent an over-familiar content, which could change their reading strategy. This also enabled systematic comparison. Although our research focused on digital content, we deliberately wanted to include paper artifacts to calibrate the digital reading habits. In contrast to the paper artifacts, the digital artifacts were brought in by the participant themselves. Since we required expertise with the format (navigation model, iconography), we asked our participants to bring their own devices with their preferred content.

Because reading is done mostly in silence and observable interaction can hardly be recorded, respondents were repeatedly asked to explain what they were currently reading, why they chose to read a certain source, and how they approached the article. In case participants

experienced too much difficulty in explaining their actions, 
we skipped this question, in order not to break the reading flow with such interruptions. On other occasions, instead, participants were encouraged to wander off, which allowed for anecdotes and personal stories, that were triggered by some articles. The latter contain a wealth of information about the related context and emotions.

The recorded sessions were afterwards analyzed with affinity diagramming: similar behavior was grouped and tagged.

This resulted in user requirements, on the basis of which we designed several paper prototypes. The latter served the discussion with the project stakeholders. After a number of iterations and corresponding refinements, a proof of concept was developed. To capture early design feedback we discussed this demonstrator with four participants (out of seven participants in total).

\section{Findings}

Moods

Our research departed from the assumption that topic selection is often hard for readers to perform accurately and unambiguously, especially because the choice to read something or not is mostly done unconsciously. Indeed, during our in-depth sessions with the research participants, we frequently observed that readers experienced difficulties when selecting preferred topics and keywords. We in particular noticed that participants often skipped entire articles, which nevertheless fully matched their areas of interest. Although the reasons behind often varied, all respondents indicated in some way that they were interested in these articles, but that they preferred to read them at a later moment, when their mood would be different. This observation provided the stepping-stone for our proposed approach. Future research will have to clarify what these moods precisely consist of, but in the current phase we defined it, in a broad and general way, as a certain feeling one has for certain kinds of information.

\begin{tabular}{|l|l|}
\hline Mode & Kind of content \\
\hline stories & $\begin{array}{l}\text { Quick and short facts. Easy to understand. } \\
\text { Clear and to-the-point. No discussions and } \\
\text { opinions. Most recent items most accessible. } \\
\text { Mixed content sources. Limited interactive } \\
\text { elements. (Similar to for example Twitter). }\end{array}$ \\
\hline $\begin{array}{l}\text { Couch } \\
\text { mode }\end{array}$ & $\begin{array}{l}\text { More elaborate articles often explaining } \\
\text { different opinions on the same subject, } \\
\text { columns, interviews, etc. Tablet optimized } \\
\text { layout supporting scanning habits. } \\
\text { Interactivity to support understanding. } \\
\text { (Presentation similar to services like Pocket } \\
\text { [8] or Instapaper [6].) }\end{array}$ \\
\hline $\begin{array}{l}\text { Inspire } \\
\text { mode }\end{array}$ & $\begin{array}{l}\text { Thought-provoking articles with a extended } \\
\text { shelf live. Content selection based on readers } \\
\text { history. Minimize distractions.(Participants } \\
\text { indicated that they did not like to fix } \\
\text { themselves to certain topics. This mode helps } \\
\text { them in discovering content.) }\end{array}$ \\
\hline $\begin{array}{l}\text { Fun and not-so-serious facts displayed in a } \\
\text { game-like environment. }\end{array}$ \\
\hline
\end{tabular}

Table 1: Overview of the different reading modes

Especially in case of digital platforms this mood based content selection can be expected to be more strongly present, compared to in the context of reading paper artifacts. In the former, readers have much more liberty in choosing the best format that matches their current mood. Moreover, on paper artifacts the presentation and content is fixed and unchangeable, while in the digital world changing the presentation of the content is often possible. 
Based on the comparison of our readers behavior, and herein inspired by insights from psychology, we could distinguish between four moods that affect reading behavior. These moods are rather abstract and in order to be selectable by our users we translated these to four reading modes: (1) latest stories mode, (2) couch mode, (3) inspiration mode and (4) play mode (see also Table 1). These modes differ in the kind of content that is presented as well as the format in which it is presented.

Personalization using context

In their discussion of reading modes, participants frequently made reference to places and time spans. Some moods were associated with certain places (for example: the couch at home) and certain times (for example: evenings). While not all modes could admittedly be directly mapped to locations and certain time spans, the connection between the reading experience and the context (i.e. time and place), was nevertheless found very useful for further content personalization.

Whereas the reading mood affects the kind of content that readers prefer, the context affects the relevance of the content. Using information on the current time and place, serves as an interesting vehicle to prioritize certain content. The particular implementation obviously also depends on the mode.

Latest stories mode: As readers want to get up-to-date with current affairs as fast as possible, time is very important in the sorting of the content. The most recent events should be the easiest to access. This mode is typically used in mobile situations (on the train, in the doctors waiting room, etc.). The current location, determined by the system, should be used to adapt the reading experience such that this mode can work as fluently as possible. For example, when having access to a high-speed connection the reading platform could try to cache as much information as possible so that reading can continue if the connection drops. Location is also important for relevant advertisements.

Couch mode: The current time affects the order of the articles but can as well influence the selection of the content. For example, many participants indicated that they like to read during lunchtime at work. Yet, such a short timespan, and a lack of attention resources, prevents them from reading long articles. These longer articles are more suited for evenings or weekends. Location hence affects the content selection in the same way as time does. Some locations are better suited for particular content. By using location estimates, we can select the most suited content and present that first to the reader.

Inspire mode: Here, time and location are much less important. Articles are retrieved based on the readers reading history. Advertisements in this mode can benefit from access to the current time, and location identification can be used to give preference to local stories, but both parameters are here less at stake.

Play mode: With the present state of our research, it is unclear how time and location could influence this mode.

№ SIM ร

\section{- 늘 1}

Figure 1: Selector for the different reading modes

Prototype

Inspired by these findings, we designed a demonstrator application. As mentioned, this helped us to gather user feedback early on. The design process of the demonstrator itself started with a paper prototyping phase 


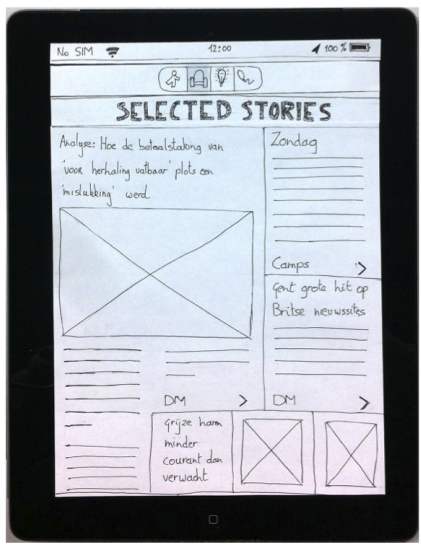

Figure 2: An example of a screen of the paper prototype (see for example Figure 3) to collect feedback from the stakeholders on the feasibility of the prototype.

The prototype uses a four button bar to select a reading mode that corresponds to the four reading moods

discussed above. Each button depicts an icon that reflects the more abstract mood. Both the content and the layout differ between the modes. In Table 1, a concise overview is given of the different modes, their content and their layout.

As suggested before, it proved not always possible to tie a reading mode to a specific time and place. Whereas linkages often exist, we consider it more reliable to make the selection of the reading mode a manual intervention. This also enables readers themselves to change their modes during a single reading session. After all, moods can quickly change.

Our participants received the demonstrator with great enthusiasm. Most of them immediately expressed their preference for a specific mode. This triggered the idea that certain modes might be mapped to certain personality types, for example as the ones described by Cacioppo [3] and Kruglanski [7].

\section{Discussion and next steps}

Present-day applications use recommender systems, mainly based on topic selection and reading histories.

Yet, this approach embodies many potential deficiencies. In contrast to this traditional method of preference selection, we suggest the use of reading mood to select the type of content. Time and place estimates can be used to further refine and personalize the content selection.
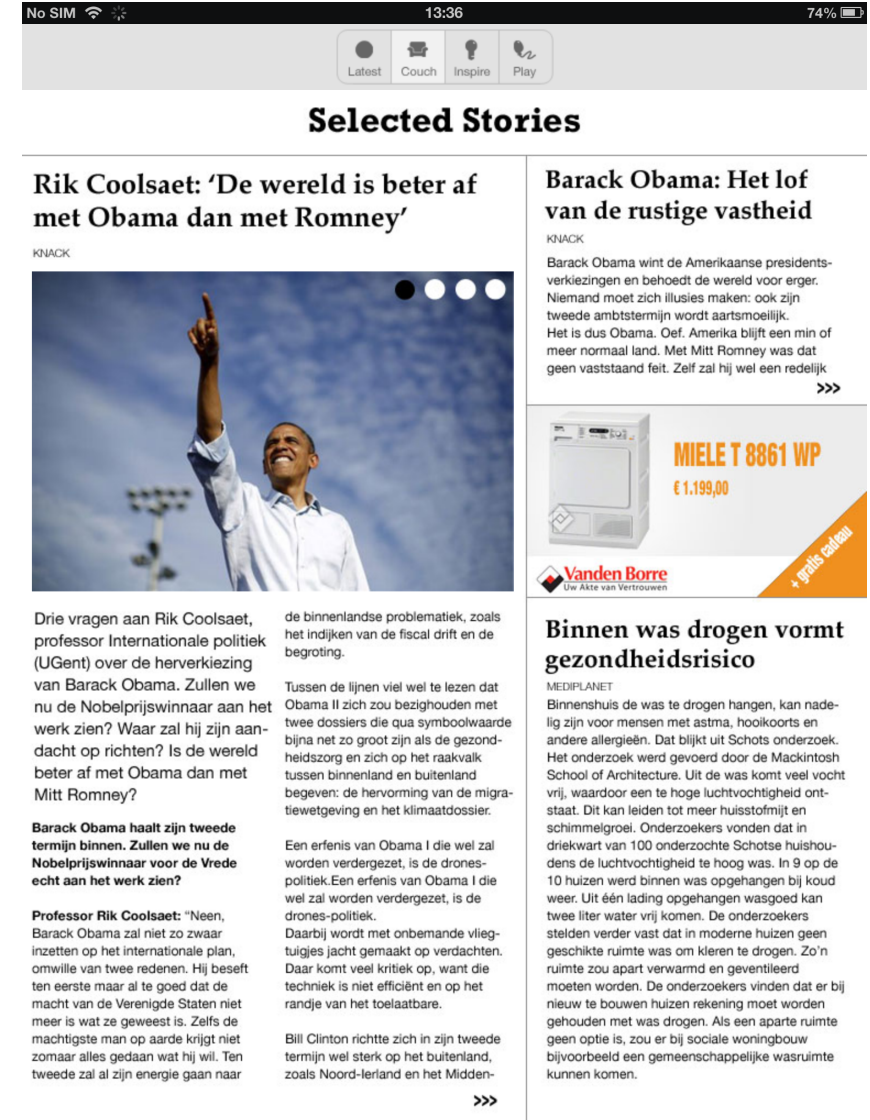

Figure 3: Couch mode in the final demonstrator

Our findings pave the way for a larger research agenda on this issue. Foremost, we need to research whether the reading modes that we derived from our observations are complete and accurate. Are there any underlying mechanisms that drive these reading modes? A more in-depth understanding of these modes is also pivotal to 
enable the writing of usable guidelines for practitioners to implement them.

In the same vein, research should investigate whether our findings can be integrated with existing recommender systems, to create contextual recommender systems. [1]

On a more theoretical note, research should assess the possibility and desirability to match our reading modes with personality typologies that are commonplace in psychology. One can think of, for instance, closure personality types of Kruglanski [7], or the cognition types of Cacioppo [3]. What remains to be more strongly investigated, also, is whether, when and how, readers switch between different modes. Experiments can hereto be useful.

Finally, our findings are limited to a very specific setting (Flanders) on the one hand, and a particular audience on the other hand (early adopters of digital reading platforms). Future research should investigate whether our insights can be extrapolated to other settings as well, and whether they hold true for other types of users.

\section{Acknowledgement}

The research leading to these results was carried out in the Smarter Media in Flanders (SMIF) project, co-funded by IWT (Agency for Innovation by Science and Technology), a Flemish government agency.

\section{References}

[1] Adomavicius, G., and Tuzhilin, A. Context-aware recommender systems. In Recommender Systems Handbook, F. Ricci, L. Rokach, B. Shapira, and P. B. Kantor, Eds. Springer US, 2011, 217-253.

[2] Beyer, H., and Holtzblatt, K. Contextual Design: Defining Customer-Centered Systems. Interactive Technologies. Elsevier Science, 1997.

[3] Cacioppo, J. T., and Petty, R. E. The need for cognition. Journal of Personality and Social Psychology 42, 1 (1982), 116-131.

[4] Currents, G. url

http://www.google.com/producer/currents.

[5] Flipboard. url http://flipboard.com.

[6] Instapaper. url http://www.instapaper.com

[7] Kruglanski, A. W., and Webster, D. M. Motivated closing of the mind: Seizing and freezing. Psychological Review 103, 2 (1996), 263-283.

[8] Pocket. url http://getpocket.com.

[9] Prismatic. url http://getprismatic.com.

[10] Zite. url http://zite.com 